\title{
PERSPECTIVE
}

\section{Enabling the Rise of a CRISPR World}

\author{
Caroline M. LaManna ${ }^{1}$ and Rodolphe Barrangou²
}

\begin{abstract}
CRISPR technology has dramatically changed scientists' ability to conduct research in medicine, biotechnology, and agriculture through faster, more efficient genome editing. A key driver of the technology's adoption is the easy, fast, and inexpensive access to vectors and the resulting next-generation tools by the nonprofit plasmid repository Addgene. Since 2013, Addgene has shipped over 100,000 CRISPR plasmids to more than 75 countries worldwide. This pipeline of new technologies is enabling cutting-edge research to address the grand challenges of mankind.
\end{abstract}

\section{Introduction}

The past decade has witnessed the rise of CRISPR * to scientific stardom, starting from the discovery of its role in adaptive immunity in bacteria ${ }^{1,2}$ to its development into a potent and accessible tool in genetics, driving advances in genome editing across medicine, biotechnology, and agriculture. ${ }^{3,4}$ Scientifically, there are many features that have enabled the rapid adoption of CRISPRbased technologies, encompassing programmability, specificity, efficiency, precision, scalability, and multiplexibility. ${ }^{5}$ There are even improvements underway that take advantage of biodiversity and engineering approaches that will further advance technological characteristics such as size, targeting flexibility, and delivery.

Another key driver of the "CRISPR Craze," however, has been the open accessibility of this technology in general, and quick and affordable access to the most cuttingedge tools in particular.

\section{Democratizing CRISPR}

Enter Addgene. What started as a nonprofit organization aimed at disseminating valuable biological material between academics has rapidly turned into the key enabler of the democratization of CRISPR technologies. ${ }^{6}$

In 2004, Melina Fan, Kenneth Fan, and Benjie Chen founded Addgene to alleviate the frustration academic

*Clustered Regularly Interspaced Short Palindromic Repeats. scientists experienced sharing plasmids with one another. The repository created a one-stop shop where scientists could deposit their frequently requested plasmids for storage and worldwide distribution-Addgene handled quality control, material transfer agreements, request tracking, and shipping logistics. As a self-sustaining nonprofit, depositing plasmids is free for scientists and a nominal fee is charged for requests, allowing for maintenance and growth of the repository without reliance on grants or external funding.

In its early years, Addgene helped scientists share cutting-edge plasmid tools such as short hairpin RNA (shRNA), lentiviral plasmids, induced pluripotent stem cell reprogramming factors, and even the CRISPR genome editing precursors (zinc finger nucleases and TALENs). As of May 2018, Addgene has shipped nearly 1 million plasmids and 6,300 ready-to-use viral vectors to scientists at more than 6,200 institutions in almost 100 countries.

In late 2012 and early 2013, CRISPR gene editing made its entrance onto the scientific scene, ${ }^{7-10}$ as well as in the Addgene repository. Leading researchers (such as Jennifer Doudna, Feng Zhang, George Church, and others) made it a priority to ensure that other labs had access to these influential tools by sharing and committing to open science. Their original CRISPR plasmids were made available immediately upon publication of the studies that have defined the field.

${ }^{1}$ Addgene, Cambridge, Massachusetts; ${ }^{2}$ Department of Food, Bioprocessing and Nutrition Sciences, North Carolina State University, Raleigh, North Carolina.

Address correspondence to: Caroline LaManna, Addgene, 75 Sidney Street, Suite 550A, Cambridge, MA 02139, E-mail: caroline@addgene.org; Rodolphe Barrangou, Department of Food, Bioprocessing and Nutrition Sciences, 400 Dan Allen Drive, Campus Box 7624, North Carolina State University, Raleigh, NC 27695, E-mail: rbarran@ncsu.edu

(C) Caroline M. LaManna and Rodolphe Barrangou 2018; Published by Mary Ann Liebert, Inc. This Open Access article is distributed under the terms of the Creative Commons Attribution Noncommercial License (http://creativecommons.org/licenses/by-nc/4.0/) which permits any noncommercial use, distribution, and reproduction in any medium, provided the original author(s) and the source are cited. 

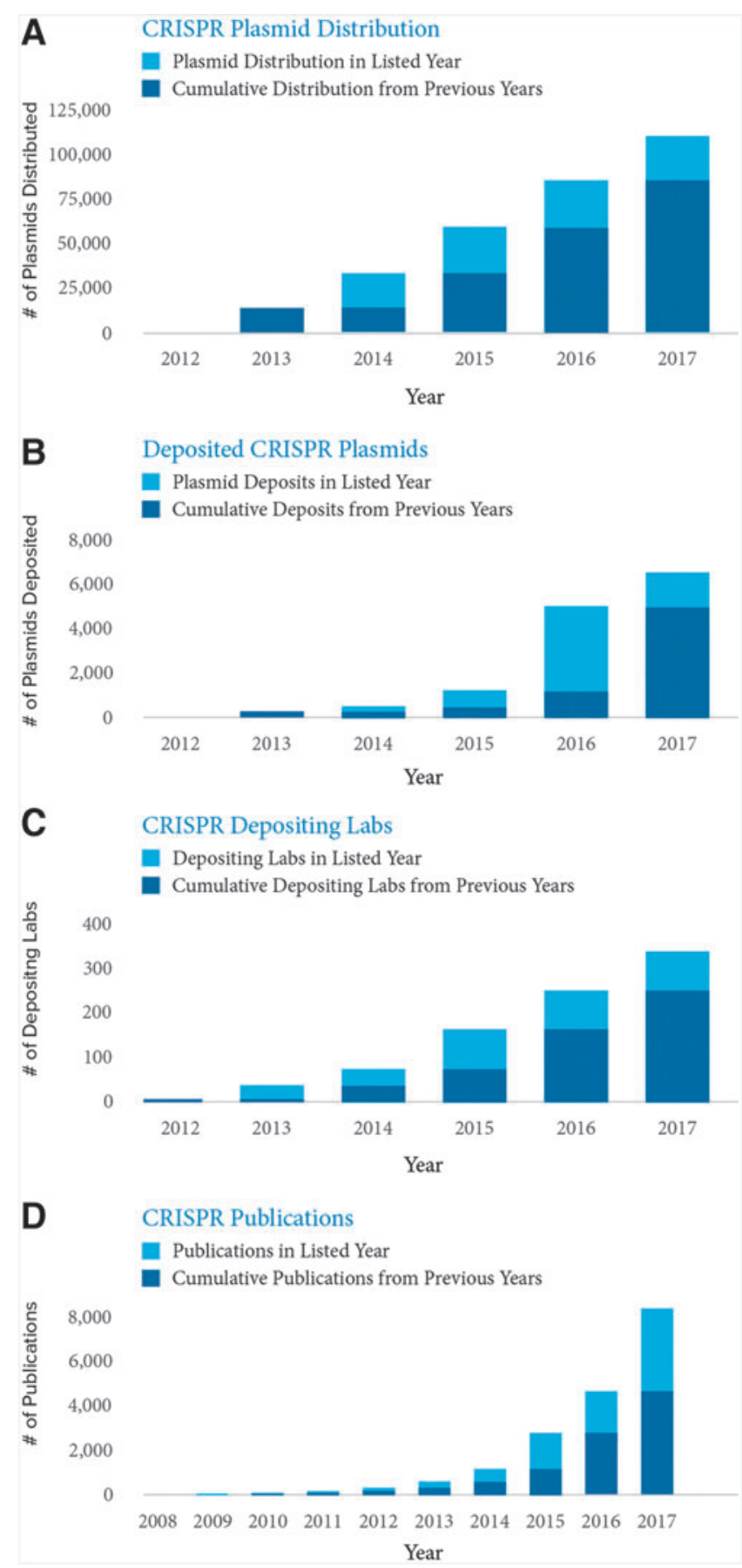

FIG. 1. Growth in adoption of CRISPR technology as shown by the number of CRISPR plasmids distributed by the Addgene plasmid repository (A), number of CRISPR plasmids deposited to Addgene (B), number of labs depositing CRISPR plasmids (C), and number of publications in PubMed linked to the keyword CRISPR (D).
In a fast-moving field in which intellectual property is a commercialization impediment and freedom to operate is a business nightmare, ${ }^{11}$ academics can request these plasmids at cost (\$65 per plasmid) and have them shipped to their lab within days. Subsequently, as scientists around the world began using these vectors, they built the next generation of CRISPR and Cas tools and deposited them with Addgene, continuing the sharing cycle and triggering further progression of the technology.

As of January 2018, Addgene has distributed more than 100,000 CRISPR plasmids to 3,400 laboratories worldwide (Fig. 1A). More than 6,300 CRISPR-related plasmids have been developed by over 330 academic labs and deposited into Addgene's collection (Fig. 1B, C). Visitors to Addgene's website have downloaded more than 30,000 copies of the CRISPR 101 ebook and viewed CRISPR blog posts over 500,000 times. ${ }^{12}$

\section{Global Reach}

Geographically, new CRISPR plasmids have been developed and deposited to Addgene's collection from the Americas (led by the United States), Europe (led by Denmark), Asia (led by China), and Oceania (led by Australia), as shown in Figure 2, and shipped to some 75 countries. Unequivocally, Addgene has driven global, fast, and affordable distribution of CRISPR technologies, helping researchers regardless of socioeconomic, geographical, or scientific status.

The considerable impact of this openly accessible material and educational resource sharing by the CRISPR community is demonstrated by the resulting publication record. Since 2008, nearly 8,000 CRISPR-related papers have been published, the majority of which were released since 2017 (Fig. 1D). These numbers are impressive, especially for an infant technology that has perhaps yet to hit its prime.

Indeed, with more academic groups contributing CRISPR-related deposits to Addgene each month-and a corresponding growth in monthly shipments-the dissemination of CRISPR is likely just beginning. Academic researchers will continue to adopt the technology but rely on Addgene for access. This will remain a key driver of CRISPR advances and be a foundation for further scientific studies, technology enhancements, and application diversification.

Importantly, the aforementioned universal access to all academics and nonprofit organizations will help equalize scientific opportunities and enable teachers at all levels to include CRISPR-related tools and applications in classrooms, ${ }^{13}$ thus enabling the education of graduates and other young scientists in real time. 


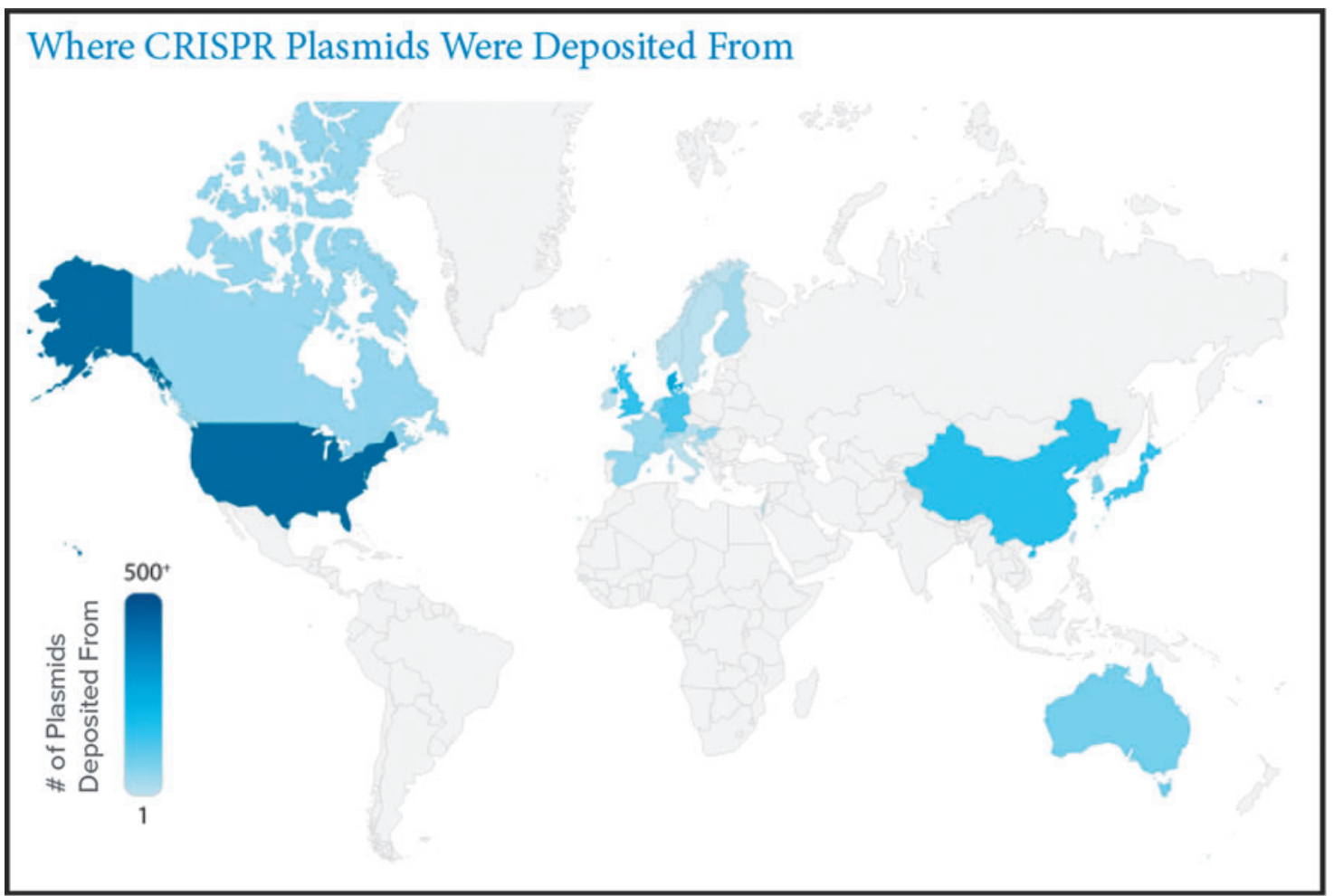

Where CRISPR Plasmids Were Distributed To
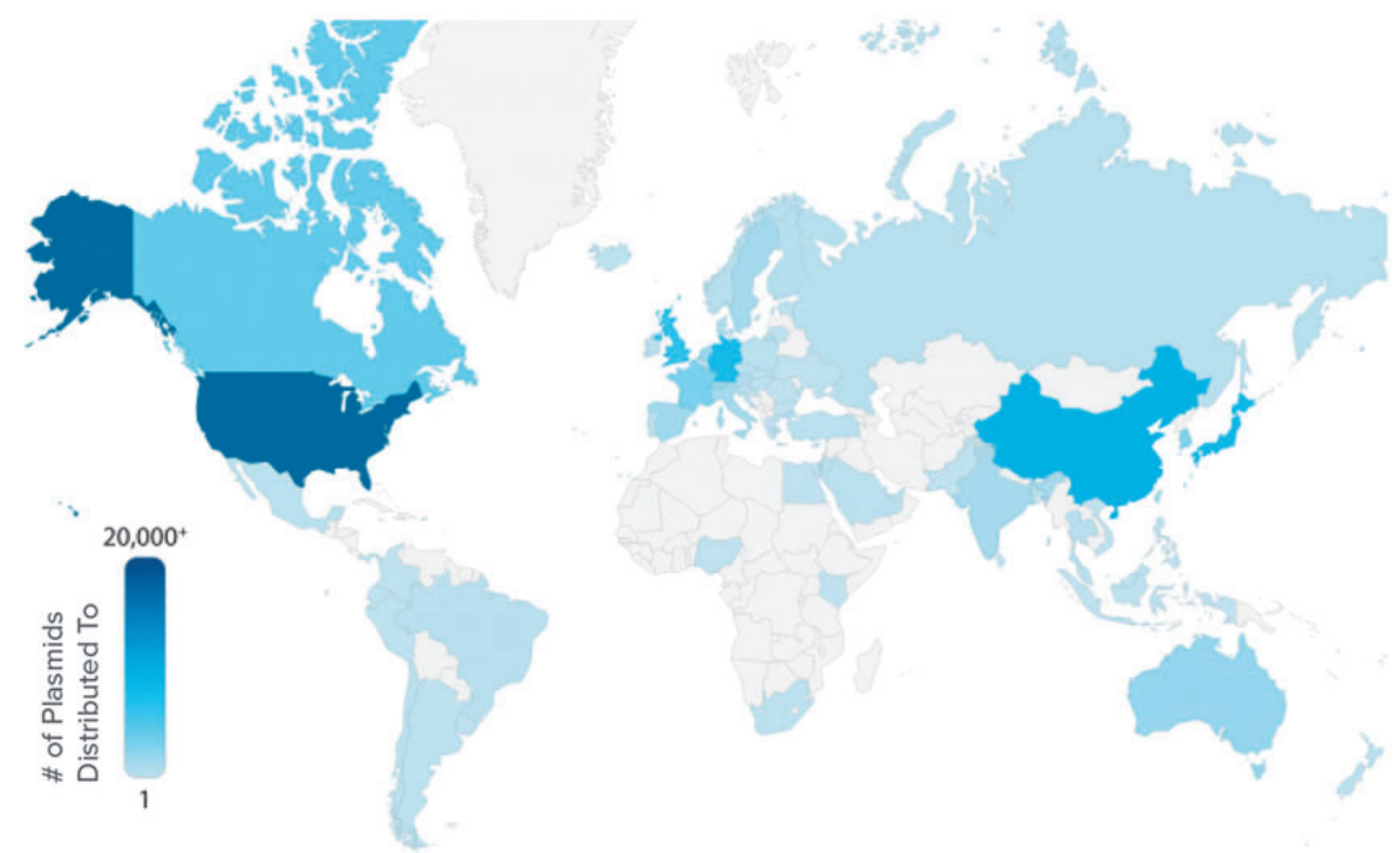

FIG. 2. Spread of CRISPR technology worldwide: (top) countries where CRISPR plasmids have been made and from which they have been deposited to Addgene; (bottom) countries to which Addgene has shipped CRISPR plasmids. The number of plasmids deposited from a country or shipped to a country are represented by colors on the maps and correspond with their respective color keys. 


\section{Conclusion}

Although society continues to debate genome editing regulations, CRISPR-based research remains pervasive in both classrooms and laboratories across the world to address the grand challenges of mankind. Addgene will continue to help scientists share their latest CRISPR advances with one another, accelerating research and enabling scientists to develop these solutions. This is, after all, what the global scientific community strives for.

\section{Acknowledgments}

R.B. and C.M.L. acknowledge support from North Carolina State University and Addgene in general, and access to shipment and deposit data in particular. We also thank Addgene's Nicole Waxmonsky for data acquisition, Wu Li for graphical assistance, and Tyler Ford for editing.

\section{Author Disclosure Statement}

C.M.L. declares no competing financial interest. R.B. is a shareholder of DuPont and Caribou Biosciences and a founder and scientific advisor to Intellia Therapeutics and Locus Biosciences.

\section{References}

1. Barrangou R, Horvath P. A decade of discovery: CRISPR functions and applications. Nat Microbiol. 2017;2:17092. DOI: 10.1038/nmicrobiol.2017.92

2. Barrangou R, Fremaux C, Deveau J, et al. CRISPR provides resistance against viruses in prokaryotes. Science. 2007;315:1709-1712.

3. Doudna JA, Charpentier E. Genome editing: the new frontier of genome engineering with CRISPR-Cas9. Science. 2014;343:1247997. DOI: 10.1126/science.1247997.

4. Hsu PD, Lander ES, Zhang F. Development and applications of CRISPRCas9 for genome engineering. Cell. 2014;157:1262-1578. DOI: 10.1016/ j.cell.2014.05.010.

5. Wright AV, Nunez JK, Doudna JA. Biology and applications of CRISPR systems: harnessing nature's toolbox for genome engineering. Cell. 2016;164:29-44. DOI: 10.1016/j.cell.2015.12.035.

6. Fan M, Tsai J, Fan K, LaBaer J. A ventral repository for published plasmids. Science. 2005;307:1877. DOI: 10.1126/science.307.5717.1877a.

7. Pennisi E. The CRISPR craze. Science. 2013;341:833-836. DOI: 10.1126/ science.341.6148.833.

8. Cong L, Ran FA, Cox D, et al. Multiplex genome engineering using CRISPR/ Cas systems. Science. 2013;339:819-823. DOI: 10.1126/science.1231143.

9. Mali $P$, Yang L, Esvelt KM, et al. RNA-guided human genome engineering via Cas9. Science. 2013;339:823-826. DOI: 10.1126/science.1232033.

10. Jinek $M$, East $A$, Lin $S$, et al. RNA-programmed genome editing in human cells. elife. 2013;2:e00471. DOI: 10.7554/eLife.00471.

11. Sherkow JS. The CRISPR patent landscape: past, present and future. CRISPR. 2018;1:5-9. DOI: 10.1089/crispr.2017.0013.

12. Patrick M. CRISPR 101: A New Series on Genome Editing \& CRISPR-Cas. Addgene, March 5, 2015. https://blog.addgene.org/crispr-101 (last accessed May 30, 2018).

13. Dahlberg L, Groat Carmona AM. CRISPR-Cas technology in and out of the classroom. CRISPR. 2018;1:107-114. DOI 10.1089/crispr.2018.0007. 